\title{
Some Properties of $p$-Groups and Commutative $p$-Groups
}

\author{
Xiquan Liang \\ Qingdao University of Science \\ and Technology \\ China
}

\author{
Dailu Li \\ Qingdao University of Science \\ and Technology \\ China
}

Summary. This article describes some properties of $p$-groups and some properties of commutative $p$-groups.

MML identifier: GROUPP_1, version: $\underline{7.11 .07 \quad 4.156 .1112}$

The notation and terminology used here have been introduced in the following papers: [7], [4], [8], [6], [10], [9], [11], [5], [1], [3], [2], and [12].

\section{1. $p$-GROUPS}

For simplicity, we use the following convention: $G$ is a group, $a, b$ are elements of $G, m, n$ are natural numbers, and $p$ is a prime natural number.

One can prove the following propositions:

(1) If for every natural number $r$ holds $n \neq p^{r}$, then there exists an element $s$ of $\mathbb{N}$ such that $s$ is prime and $s \mid n$ and $s \neq p$.

(2) For all natural numbers $n, m$ such that $n \mid p^{m}$ there exists a natural number $r$ such that $n=p^{r}$ and $r \leq m$.

(3) If $a^{n}=\mathbf{1}_{G}$, then $\left(a^{-1}\right)^{n}=\mathbf{1}_{G}$.

(4) If $\left(a^{-1}\right)^{n}=\mathbf{1}_{G}$, then $a^{n}=\mathbf{1}_{G}$.

(5) $\operatorname{ord}\left(a^{-1}\right)=\operatorname{ord}(a)$.

(6) $\operatorname{ord}\left(a^{b}\right)=\operatorname{ord}(a)$.

(7) Let $G$ be a group, $N$ be a subgroup of $G$, and $a, b$ be elements of $G$. Suppose $N$ is normal and $b \in N$. Let given $n$. Then there exists an element $g$ of $G$ such that $g \in N$ and $(a \cdot b)^{n}=a^{n} \cdot g$. 
(8) Let $G$ be a group, $N$ be a normal subgroup of $G, a$ be an element of $G$, and $S$ be an element of $G / N$. If $S=a \cdot N$, then for every $n$ holds $S^{n}=a^{n} \cdot N$.

(9) Let $G$ be a group, $H$ be a subgroup of $G$, and $a, b$ be elements of $G$. If $a \cdot H=b \cdot H$, then there exists an element $h$ of $G$ such that $a=b \cdot h$ and $h \in H$.

(10) Let $G$ be a finite group and $N$ be a normal subgroup of $G$. If $N$ is a subgroup of $\mathrm{Z}(G)$ and ${ }^{G} / N$ is cyclic, then $G$ is commutative.

(11) Let $G$ be a finite group and $N$ be a normal subgroup of $G$. If $N=\mathrm{Z}(G)$ and ${ }^{G} / N$ is cyclic, then $G$ is commutative.

(12) For every finite group $G$ and for every subgroup $H$ of $G$ such that $\overline{\bar{H}} \neq \overline{\bar{G}}$ there exists an element $a$ of $G$ such that $a \notin H$.

Let $p$ be a natural number, let $G$ be a group, and let $a$ be an element of $G$. We say that $a$ is $p$-power if and only if:

(Def. 1) There exists a natural number $r$ such that $\operatorname{ord}(a)=p^{r}$.

We now state the proposition

(13) $\mathbf{1}_{G}$ is $m$-power.

Let us consider $G, m$. One can verify that there exists an element of $G$ which is $m$-power.

Let us consider $p, G$ and let $a$ be a $p$-power element of $G$. Observe that $a^{-1}$ is $p$-power.

One can prove the following proposition

(14) If $a^{b}$ is $p$-power, then $a$ is $p$-power.

Let us consider $p, G, b$ and let $a$ be a $p$-power element of $G$. One can verify that $a^{b}$ is $p$-power.

Let us consider $p$, let $G$ be a commutative group, and let $a, b$ be $p$-power elements of $G$. Observe that $a \cdot b$ is $p$-power.

Let us consider $p$ and let $G$ be a finite $p$-group group. One can verify that every element of $G$ is $p$-power.

The following proposition is true

(15) Let $G$ be a finite group, $H$ be a subgroup of $G$, and $a$ be an element of $G$. If $H$ is $p$-group and $a \in H$, then $a$ is $p$-power.

Let us consider $p$ and let $G$ be a finite $p$-group group. One can verify that every subgroup of $G$ is $p$-group.

We now state the proposition

(16) $\{\mathbf{1}\}_{G}$ is $p$-group.

Let us consider $p$ and let $G$ be a group. Note that there exists a subgroup of $G$ which is $p$-group. 
Let us consider $p$, let $G$ be a finite group, let $G_{1}$ be a $p$-group subgroup of $G$, and let $G_{2}$ be a subgroup of $G$. One can verify that $G_{1} \cap G_{2}$ is p-group and $G_{2} \cap G_{1}$ is $p$-group.

Next we state the proposition

(17) For every finite group $G$ such that every element of $G$ is $p$-power holds $G$ is $p$-group.

Let us consider $p$, let $G$ be a finite $p$-group group, and let $N$ be a normal subgroup of $G$. Note that $G /{ }_{N}$ is $p$-group.

The following four propositions are true:

(18) Let $G$ be a finite group and $N$ be a normal subgroup of $G$. If $N$ is $p$-group and $G / N$ is $p$-group, then $G$ is $p$-group.

(19) Let $G$ be a finite commutative group and $H, H_{1}, H_{2}$ be subgroups of $G$. Suppose $H_{1}$ is $p$-group and $H_{2}$ is $p$-group and the carrier of $H=H_{1} \cdot H_{2}$. Then $H$ is $p$-group.

(20) Let $G$ be a finite group and $H, N$ be subgroups of $G$. Suppose $N$ is a normal subgroup of $G$ and $H$ is $p$-group and $N$ is $p$-group. Then there exists a strict subgroup $P$ of $G$ such that the carrier of $P=H \cdot N$ and $P$ is $p$-group.

(21) Let $G$ be a finite group and $N_{1}, N_{2}$ be normal subgroups of $G$. Suppose $N_{1}$ is $p$-group and $N_{2}$ is $p$-group. Then there exists a strict normal subgroup $N$ of $G$ such that the carrier of $N=N_{1} \cdot N_{2}$ and $N$ is $p$-group.

Let us consider $p$, let $G$ be a $p$-group finite group, let $H$ be a finite group, and let $g$ be a homomorphism from $G$ to $H$. Observe that $\operatorname{Im} g$ is $p$-group.

The following proposition is true

(22) For all strict groups $G, H$ such that $G$ and $H$ are isomorphic and $G$ is $p$-group holds $H$ is $p$-group.

Let $p$ be a prime natural number and let $G$ be a group. Let us assume that $G$ is $p$-group. The functor expon $(G, p)$ yields a natural number and is defined by:

(Def. 2) $\overline{\bar{G}}=p^{\operatorname{expon}(G, p)}$.

Let $p$ be a prime natural number and let $G$ be a group. Then $\operatorname{expon}(G, p)$ is an element of $\mathbb{N}$.

Next we state four propositions:

(23) For every finite group $G$ and for every subgroup $H$ of $G$ such that $G$ is $p$-group holds expon $(H, p) \leq \operatorname{expon}(G, p)$.

(24) For every strict finite group $G$ such that $G$ is $p$-group and $\operatorname{expon}(G, p)=$ 0 holds $G=\{\mathbf{1}\}_{G}$.

(25) For every strict finite group $G$ such that $G$ is $p$-group and $\operatorname{expon}(G, p)=$ 1 holds $G$ is cyclic. 
(26) Let $G$ be a finite group, $p$ be a prime natural number, and $a$ be an element of $G$. If $G$ is $p$-group and expon $(G, p)=2$ and $\operatorname{ord}(a)=p^{2}$, then $G$ is commutative.

\section{Commutative $p$-Groups}

Let $p$ be a natural number and let $G$ be a group. We say that $G$ is $p$ commutative group-like if and only if:

(Def. 3) For all elements $a, b$ of $G$ holds $(a \cdot b)^{p}=a^{p} \cdot b^{p}$.

Let $p$ be a natural number and let $G$ be a group. We say that $G$ is $p$ commutative group if and only if:

(Def. 4) $G$ is $p$-group and $p$-commutative group-like.

Let $p$ be a natural number. Observe that every group which is $p$-commutative group is also $p$-group and $p$-commutative group-like and every group which is $p$-group and $p$-commutative group-like is also $p$-commutative group.

The following proposition is true

(27) $\{\mathbf{1}\}_{G}$ is $p$-commutative group-like.

Let us consider $p$. Note that there exists a group which is $p$-commutative group, finite, cyclic, and commutative.

Let us consider $p$ and let $G$ be a $p$-commutative group-like finite group. Note that every subgroup of $G$ is $p$-commutative group-like.

Let us consider $p$. Note that every group which is $p$-group, finite, and commutative is also $p$-commutative group.

We now state the proposition

(28) For every strict finite group $G$ such that $\overline{\bar{G}}=p$ holds $G$ is $p$-commutative group.

Let us consider $p, G$. One can check that there exists a subgroup of $G$ which is $p$-commutative group and finite.

Let us consider $p$, let $G$ be a finite group, let $H_{1}$ be a $p$-commutative grouplike subgroup of $G$, and let $H_{2}$ be a subgroup of $G$. One can check that $H_{1} \cap H_{2}$ is $p$-commutative group-like and $H_{2} \cap H_{1}$ is $p$-commutative group-like.

Let us consider $p$, let $G$ be a finite $p$-commutative group-like group, and let $N$ be a normal subgroup of $G$. One can verify that $G / N$ is $p$-commutative group-like.

One can prove the following propositions:

(29) Let $G$ be a finite group and $a, b$ be elements of $G$. Suppose $G$ is $p$ commutative group-like. Let given $n$. Then $(a \cdot b)^{p^{n}}=a^{p^{n}} \cdot b^{p^{n}}$.

(30) Let $G$ be a finite commutative group and $H, H_{1}, H_{2}$ be subgroups of $G$. Suppose $H_{1}$ is $p$-commutative group and $H_{2}$ is $p$-commutative group and the carrier of $H=H_{1} \cdot H_{2}$. Then $H$ is $p$-commutative group. 
(31) Let $G$ be a finite group, $H$ be a subgroup of $G$, and $N$ be a strict normal subgroup of $G$. Suppose $N$ is a subgroup of $\mathrm{Z}(G)$ and $H$ is $p$-commutative group and $N$ is $p$-commutative group. Then there exists a strict subgroup $P$ of $G$ such that the carrier of $P=H \cdot N$ and $P$ is $p$-commutative group.

(32) Let $G$ be a finite group and $N_{1}, N_{2}$ be normal subgroups of $G$. Suppose $N_{2}$ is a subgroup of $\mathrm{Z}(G)$ and $N_{1}$ is $p$-commutative group and $N_{2}$ is $p$ commutative group. Then there exists a strict normal subgroup $N$ of $G$ such that the carrier of $N=N_{1} \cdot N_{2}$ and $N$ is $p$-commutative group.

(33) Let $G, H$ be groups. Suppose $G$ and $H$ are isomorphic and $G$ is $p$ commutative group-like. Then $H$ is $p$-commutative group-like.

(34) Let $G, H$ be strict groups. Suppose $G$ and $H$ are isomorphic and $G$ is $p$-commutative group. Then $H$ is $p$-commutative group.

Let us consider $p$, let $G$ be a $p$-commutative group-like finite group, let $H$ be a finite group, and let $g$ be a homomorphism from $G$ to $H$. Observe that $\operatorname{Im} g$ is $p$-commutative group-like.

The following propositions are true:

(35) For every strict finite group $G$ such that $G$ is $p$-group and $\operatorname{expon}(G, p)=$ 0 holds $G$ is $p$-commutative group.

(36) For every strict finite group $G$ such that $G$ is $p$-group and $\operatorname{expon}(G, p)=$ 1 holds $G$ is $p$-commutative group.

\section{REFERENCES}

[1] Grzegorz Bancerek. The fundamental properties of natural numbers. Formalized Mathematics, 1(1):41-46, 1990.

[2] Grzegorz Bancerek. The ordinal numbers. Formalized Mathematics, 1(1):91-96, 1990.

[3] Rafał Kwiatek. Factorial and Newton coefficients. Formalized Mathematics, 1(5):887-890, 1990.

[4] Marco Riccardi. The Sylow theorems. Formalized Mathematics, 15(3):159-165, 2007, doi:10.2478/v10037-007-0018-3.

[5] Dariusz Surowik. Cyclic groups and some of their properties - part I. Formalized Mathematics, 2(5):623-627, 1991.

[6] Wojciech A. Trybulec. Classes of conjugation. Normal subgroups. Formalized Mathematics, 1(5):955-962, 1990.

[7] Wojciech A. Trybulec. Groups. Formalized Mathematics, 1(5):821-827, 1990.

[8] Wojciech A. Trybulec. Subgroup and cosets of subgroups. Formalized Mathematics, $1(5): 855-864,1990$.

[9] Wojciech A. Trybulec. Commutator and center of a group. Formalized Mathematics, 2(4):461-466, 1991

[10] Wojciech A. Trybulec. Lattice of subgroups of a group. Frattini subgroup. Formalized Mathematics, 2(1):41-47, 1991.

[11] Wojciech A. Trybulec and Michał J. Trybulec. Homomorphisms and isomorphisms of groups. Quotient group. Formalized Mathematics, 2(4):573-578, 1991.

[12] Zinaida Trybulec. Properties of subsets. Formalized Mathematics, 1(1):67-71, 1990. 